\title{
Counting grains of sound
}

Sound and vibration come in discrete units called phonons, in the same way that light exists as photons. A superconducting device that can count phonons could lead to advances in quantum-information processing. SEE LETTER P.537

\section{ALBERT SCHLIESSER}

A ccording to a central principle of quantum mechanics, the energy of a physical system is quantized - energy can be increased or decreased only in discrete steps. For example, sound and vibration are quantized in discrete packets called phonons. On page 537, Arrangoiz-Arriola et al. ${ }^{1}$ report a superconducting device that can directly indicate the number of phonons present in the vibration of a nanometre-scale structure. This work not only demonstrates the quantization of vibrational energy, but also provides a handle on the quantum states of phonons that could enable innovative tools for quantuminformation processing.

Countless experiments in quantum optics have confirmed the existence and properties of photons (packets of light), in a spectacularly successful application of quantum theory. Today, techniques abound to manipulate and detect light at the level of individual photons, which in turn form the basis of many quantum technologies. The situation has been somewhat different for sound and vibration. The existence of phonons has long been recognized, and underlies our understanding of many properties of solids. But technology to measure and control vibrations at the quantum level is still in its infancy.

Arrangoiz-Arriola and colleagues follow an approach known as quantum acoustics ${ }^{2-4}$ (the acoustic analogue of quantum optics), in which a vibrating structure is coupled to an artificial atom. This atom exists in one of two states, and the absorption of a single phonon from the vibrating structure is sufficient to induce a transition between the states. As a result, methods to detect and prepare the state of the atom allow phonons to be controlled one by one.

In the authors' work, the artificial atom is a transmon qubit - a sophisticated superconducting circuit that operates at microwave frequencies. Technical details aside, these devices are hailed as potential building blocks of future quantum computers, and mature techniques exist to measure and manipulate their state. For quantum acoustics, it is essential to achieve the largest possible coupling between the transmon qubit and the vibrating structure.

Arrangoiz-Arriola et al. manage to achieve
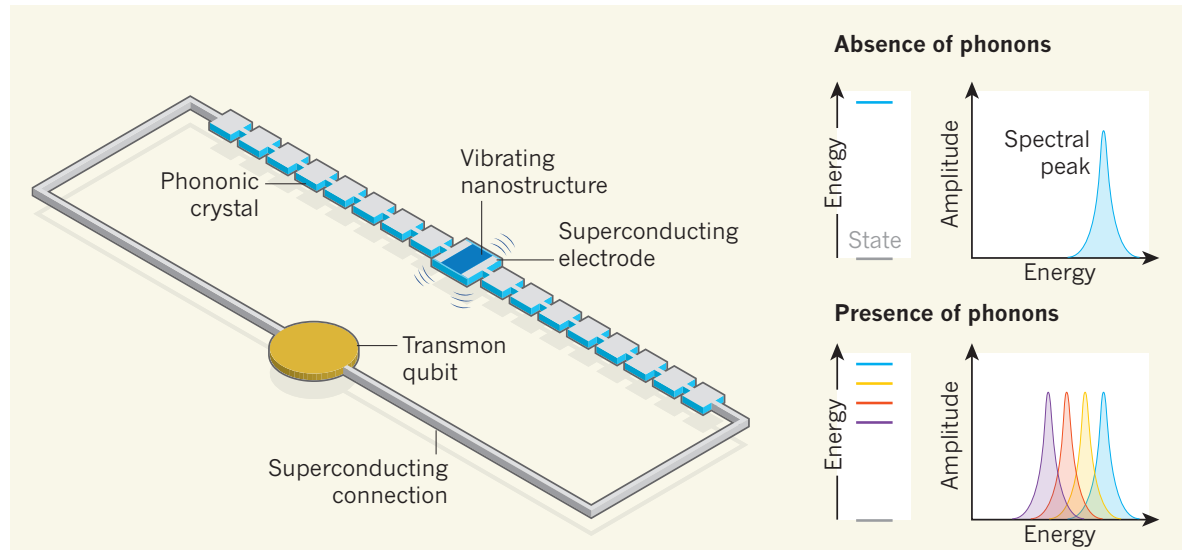

Presence of phonons

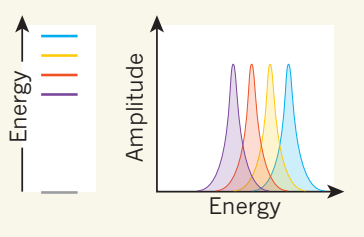

Figure 1 | A phonon-counting platform. a, Arrangoiz-Arriola et al. ${ }^{1}$ report a way to determine how many phonons (quantum units of vibrational energy) are present in a vibrating nanostructure. The authors link the nanostructure to a superconducting device called a transmon qubit using superconducting electrodes and connections. They use a structure known as a phononic crystal (light blue layer) to reduce loss of phonons to the environment. b. The transmon qubit exists in one of two states. In the absence of phonons, a transition between these states requires a specific energy and produces a single spectral peak. The presence of phonons in the vibrating nanostructure causes the transition energy and the spectral peak to shift by a discrete amount. In the example shown, there are peaks corresponding to zero (blue), one (yellow), two (red) and three (purple) phonons. Quantum mechanics allows different numbers of phonons to exist simultaneously, which gives rise to several peaks.

a coupling so strong that it is no longer necessary for a phonon to be absorbed by the qubit to be detected. Rather, the joint system can be operated in a parameter range known as the dispersive regime, in which the mere presence of a single phonon shifts the energy required to change the state of the qubit by a discrete amount $^{5}$. This energy shift is measurable because it is much larger than the uncertainty in the energy difference between the two states. Therefore, in a spectroscopy experiment, the spectral peak that is associated with the transition of the qubit from one state to the other shifts by an amount that is larger than its linewidth (a measure of the peak's width).

The authors observed the appearance of such a shifted peak when a weak stimulus excited a nanostructure and caused it to vibrate. This feature indicates the presence of a single phonon. As the amplitude of the stimulus was increased, further peaks appeared, signalling the presence of two phonons, three phonons, and so on. Quantum mechanics enables different numbers of phonons to exist at the same time, so that multiple peaks are seen. These findings are in disagreement with the classical (non-quantum) understanding of sound, which would suggest that the spectral peak shifts continuously, by an amount proportional to the average vibrational energy of the nanostructure. Instead, the data reveal the quantization of vibrational energy with striking clarity.

Clever engineering of the vibrating nanostructure is crucial to the success of the authors' phonon-counting platform (Fig. 1). First, the nanostructure is fabricated from lithium niobate, which is strongly piezoelectric it produces a large voltage in response to mechanical deformation. As a result, the nanostructure's motion is accompanied by relatively large electric fields, to which the qubit is susceptible. Second, as an innovation in quantum acoustics, the authors pattern the lithium niobate into a structure known as a phononic crystal, in which the speed of sound is periodically modulated. This crystal shields the joint system from detrimental effects of the environment, and allows only one type of phonon to interact with the qubit, which yields a clean signal.

Although the phononic crystal reduces loss of phonons to the environment, this loss is still 
a limiting factor. Qubit spectroscopy takes time - in general, more time is required when the linewidth of the peak needs to be narrow. Phonons are lost during this time, and so the probability that a particular number of phonons is present in a vibration changes as the measurement proceeds. This loss also limits the number of phonons for which simultaneous presence can be evidenced.

Results presented this year using silicon phononic crystals ${ }^{6}$ suggest that phonon loss could be further reduced in ArrangoizArriola and colleagues' platform. It might then be possible to carry out quantum nondemolition measurements of vibrational energy; these would reveal the number of phonons without changing it, so that repeated measurements yield the same result. This is a long-held dream of researchers concerned with measuring mechanical systems, because it embodies many of the fundamental principles of quantum measurements.

The technological potential of the authors' platform is just as exciting. One possible application is in a quantum modem, which is needed to realize networks of quantum computers that are in different locations. Such a modem would link superconducting qubits to optical photons (which can travel along fibre-optic networks) through a cascaded interface, whereby the qubit-phonon coupling presented here is combined with phonon-photon links developed in the field of cavity optomechanics ${ }^{7}$. And finally, a whole new architecture for quantum computers could potentially emerge, in which superconducting qubits process information stored in compact phonon registers - perhaps a more speculative prospect, but certainly an intriguing one.

Albert Schliesser is at the Niels Bohr Institute, University of Copenhagen, 2100 Copenhagen, Denmark. e-mail:albert.schliesser@nbi.ku.dk

1. Arrangoiz-Arriola, P. et al. Nature $\mathbf{5 7 1}, \mathbf{5 3 7 - 5 4 0}$ (2019).

2. O'Connell, A. D. et al. Nature $464,697-703$ (2010).

3. Chu, Y. et al. Science 358, 199-202 (2017)

4. Satzinger, K. J. et al. Nature 563, 661-665 (2018)

5. Sletten, L. R., Moores, B. A., Viennot, J. J. \& Lehnert, K. W. Phys. Rev. X 9, 021056 (2019).

6. MacCabe, G. S. et al. Preprint at https://arxiv.org/ abs/1901.04129 (2019).

7. Aspelmeyer, M., Kippenberg, T. J. \& Marquardt, F. Rev. Mod. Phys. 86, 1391-1452 (2014).

\section{Lack of PINK1 protein studied in mice}

In mice lacking a protein genetically linked to Parkinson's disease, an autoimmune response to gut infection compromises the function of dopamine-producing neurons and leads to transient movement impairments. SEE LETTER P.565

\section{MARY K. HERRICK \& MALÚ G. TANSEY}

$\mathrm{P}$ arkinson's disease is a brain disorder characterized by problems with movement and by a loss of neuronal cells in the substantia nigra of the midbrain that produce the neurotransmitter dopamine. Its causes are not clear, although some evidence suggests that disrupted immune function and inflammation, possibly in the gut, might have a part to play. Two proteins, PINK1 and parkin, regulate the function of energy-generating organelles in the cell called mitochondria ${ }^{1-3}$, and are dysfunctional in certain forms of Parkinson's disease. Matheoud et al. ${ }^{4}$ show on page 565 that, in mice lacking PINK1, an intestinal infection can trigger an immune response that results in the production of immune cells that target mitochondrial molecules. This response also causes transient motor impairments resembling those in Parkinson's disease and a temporary loss of neuronal dopaminerelease sites.

Mutations in the genes encoding PINK1 and parkin have been linked to rare, heritable members of parkinsonian syndromes ${ }^{5-7}$, the group of disorders to which Parkinson's disease belongs. However, these proteins were long considered mostly irrelevant for the approximately $90 \%$ of cases of Parkinson's disease that occur later in life (known as idiopathic Parkinson's disease), and which are thought to arise from complex interactions between genes and

the environment ${ }^{8}$.

PINK1 and parkin act together in stressed cells to protect mitochondrial function. In their absence, damaged mitochondria do not undergo proper degradation and instead accumulate in the cell ${ }^{9-11}$. Findings in the past decade or so suggest that PINK1 and parkin also have a role in immune function that could implicate them in the development of idiopathic Parkinson's disease. For example, PINK1 limits the production of inflammatory molecules called cytokines ${ }^{12}$, and parkin protects mice

\section{"Infected} mutant mice showed a loss of dopaminerelease sites in the striatum of the brain." against neurodegeneration that is caused by chronic inflammation outside the nervous system ${ }^{13}$.

In 2016, researchers from the same group as Matheoud et al. reported that PINK1 and parkin affect another immune process, known as antigen presentation - in which protein fragments called antigens are displayed on the surface of cells to signal to the immune system $^{14}$. More specifically, the researchers observed that, in cultured cells, the two proteins suppress the presentation of antigens derived from degraded mitochondria (mitochondrial-antigen presentation) that is induced by exposure to lipopolysaccharide molecules components of the outer cell membranes of the Gram-negative group of bacteria.

Matheoud et al. now extend these findings in mice that lack the gene encoding PINK1 (Pink1-knockout mice). When they exposed antigen-presenting cells called dendritic cells from these mice to various Gram-negative bacteria that commonly infect the gut, they observed that mitochondrial-antigen presentation was induced in these cells (Fig. 1a). However, this did not happen with exposure to Gram-positive bacteria, which lack lipopolysaccharides in their cell membranes.

The authors found that Pink1-knockout mice infected with the Gram-negative bacterium Citrobacter rodentium showed a loss of dopamine-release sites in the striatum of the brain, as well as motor deficits similar to those seen in Parkinson's disease (including reductions in overall movement and in motor coordination). The motor deficits were reversed by treatment with L-DOPA, the precursor to dopamine that is frequently used to treat individuals who have Parkinson's disease. One year after infection, the motor deficits and reductions in dopamine-release sites had reversed without any treatment, implying that these effects were transient. The authors did not, however, analyse whether the mice exhibited non-motor symptoms related to Parkinson's disease, such as increases in the permeability of the gut wall and in the time taken for material to pass through the digestive system. Such analyses would have strengthened the overall study.

Dendritic cells present antigens to train other immune cells known as cytotoxic $T$ cells to kill cells elsewhere in the body that present the same antigen in complex with a protein called a major histocompatibility complex (MHC) class I molecule. Matheoud et al. showed that, when cultured with a mitochondrial antigen, immune cells from the spleens of infected Pink1-knockout mice (but not from those of infected mice that expressed PINK1) generated cytotoxic $\mathrm{T}$ cells specific for the mitochondrial antigen (Fig. 1b). Dopamine neurons can upregulate surface expression 